\title{
NEIGHBORHOODS OF THE DIAGONAL AND STRONG NORMALITY PROPERTIES
}

\author{
H. L. SHAPIRO AND F. A. SMITH
}

ABSTRACT. We define the concept of $n$-even and $\aleph_{0}$-even covers and show their relationship to collectionwise normality and normality.

A completely regular topological space is said to be strongly collectionwise normal if the universal uniformity on $X$ is equal to the collection of all neighborhoods of the diagonal.

It is known that if $X$ is paracompact then $X$ is strongly collectionwise normal and that strongly collectionwise normality implies collectionwise normality (see [2] and [3]). Furthermore, in general neither of these implications can be reversed.

In this note we introduce three types of families using neighborhoods of the diagonal of $X$. We then use these to characterize strongly collectionwise normal, collectionwise noral and normal spaces.

DEFINITION. If $X$ is a topological space and if $n \in N$, we say that a cover $\mathcal{G}$ is $n$-even if there exist neighborhoods $W_{1}, \ldots, W_{n}$ of the diagonal of $X$ such that $W_{i}^{2} \subset W_{i-1}$ for $i=2, \ldots, n$ and $\left(W_{1}(x)\right)_{x \in X}$ refines $\mathcal{G}$. If there exists a sequence $\left(W_{i}\right)_{i \in N}$ of neighborhoods of the diagonal of $X$ such that $W_{i}^{2} \subset$ $W_{i-1}$ for all $n \in N, n \neq 1$, and $\left(W_{1}(x)\right)_{x \in X}$ refines $\mathcal{G}$ we say that $\mathcal{G}$ is $\aleph_{0}$-even. If $n=1$, we write "even" instead of " 1 -even" and note that this is the usual definition of even.

A sequence $\left(\mathscr{U}_{n}\right)_{n \in N}$ of open covers of a topological space $X$ is normal if for all $n \in N, \mathscr{Q}_{n+1}^{*}$ refines $\mathscr{Q}_{n}$ (i.e., $\left(\operatorname{st}\left(U, \mathscr{Q}_{n+1}\right)\right)_{U \in \mathscr{U}_{n+1}}$ refines $\left.\mathscr{U}_{n}\right)$. The cover $\mathcal{G}$ is normal if there is a normal sequence $\left(\mathscr{U}_{n}\right)_{n \in N}$ of open covers of $X$ such that $\mathcal{Q}_{1}$ refines $\mathcal{G}$.

In [4] we proved the following two results.

THEOREM 1. If $\mathcal{G}$ is an open cover of a topological space $X$ then $\mathcal{G}$ is normal if and only if $\mathcal{G}$ is $\boldsymbol{x}_{0}$-even.

THEOREM 2. A completely regular topological space is strongly collectionwise normal if and only if every even open cover is normal.

DEFINITION. Let $\mathcal{G}=\left(G_{\alpha}\right)_{\alpha \in I}$ be a family of subsets of a topological space $X$. We say that $\mathcal{G}$ is weakly even if there exists a neighborhood $W$ of the

Presented to the Society, June 4, 1978; received by the editors June 14, 1977 and, in revised form, October 24, 1977.

AMS (MOS) subject classifications (1970). Primary 54D15.

๑) American Mathematical Society 1978 
diagonal of $X$ such that $W^{2} \subset \cup_{\alpha \in I}\left(G_{\alpha} \times G_{\alpha}\right)$. We say that $\mathcal{G}$ is evenscreenable if there exist an open family $\mathscr{F}=\left(F_{\alpha}\right)_{\alpha \in I}$ and a neighborhood $W$ of the diagonal of $X$ such that $\cup \mathscr{F}=\cup \mathcal{G}$ and $W\left(F_{\alpha}\right) \subset G_{\alpha}$ for all $\alpha \in I$. The family $\mathcal{G}$ is even-expandable if there exist a locally finite family $\mathcal{H}=$ $\left(H_{\alpha}\right)_{\alpha \in I}$ and a neighborhood $W$ of the diagonal of $X$ such that $W\left(G_{\alpha}\right) \subset H_{\alpha}$ for all $\alpha \in I$.

First let us show that an even cover of a topological space is weakly even. Then let us use weakly even to characterize strongly collectionwise normal and normal spaces.

Proposition 3. If $\mathcal{G}=\left(G_{\alpha}\right)_{\alpha \in I}$ is an even cover of a topological space $X$ then $\mathcal{G}$ is a weakly even cover of $X$.

Proof. If $\mathcal{G}$ is an even cover of $X$ then there exists an open symmetric neighborhood $W$ of the diagonal of $X$ such that $(W(x))_{x \in X}$ refines $\mathcal{G}$. It is easily seen that $W^{2} \subset \cup_{\alpha \in I}\left(G_{\alpha} \times G_{\alpha}\right)$.

THEOREM 4. If $X$ is a completely regular topological space then the following statements are equivalent:

(1) The space $X$ is strongly collectionwise normal.

(2) Every open cover of $X$ is weakly even.

Proof. (1) implies (2). If $\mathcal{G}=\left(G_{\alpha}\right)_{\alpha \in I}$ is an open cover of $X$ then $W=$ $\cup_{\alpha \in I}\left(G_{\alpha} \times G_{\alpha}\right)$ is a neighborhood of the diagonal of $X$ and, therefore, $W$ is an element of the universal uniformity $\mathscr{U}_{0}$. Hence there exists an open symmetric element $U$ in $\mathscr{U}_{0}$ such that $U^{2} \subset W$. It follows that $\mathcal{G}$ is weakly even.

(2) implies (1). Clearly the universal uniformity is contained in the collection of all neighborhoods of the diagonal. On the other hand, suppose that $W$ is an open neighborhood of the diagonal of $X$ and let $\mathscr{W}=$ $(W(x))_{x \in X}$. Then $\mathscr{W}$ is an open cover of $X$ and, therefore, by (2) $\mathscr{W}$ is weakly even so there exists an open symmetric neighborhood $U_{1}$ of the diagonal of $X$ such that $U_{1}^{2} \subset \cup_{x \in X}(W(x) \times W(x))$. Let $\mathscr{U}_{1}=\left(U_{1}(x)\right)_{x \in X}$ and observe that $\left(\operatorname{st}\left(x, \mathscr{U}_{1}\right)\right)_{x \in X}$ refines $\mathscr{W}$. Furthermore, $\mathscr{U}_{1}$ is an open cover of $X$ and hence by hypothesis there exists an open symmetric neighborhood $U_{2}$ of the diagonal of $X$ such that $U_{2}^{2} \subset U_{x \in X}\left(U_{1}(x) \times U_{1}(x)\right)$. Continuing inductively we obtain a sequence of open covers $\left(\mathscr{U}_{n}\right)_{n \in N}$ such that $\left(\operatorname{st}\left(x, \mathscr{U}_{n}\right)\right)_{x \in X}$ refines $\mathcal{U}_{n-1}$ for each $n \in N, n \neq 1$. It follows that $\left(\mathscr{Q}_{2 n}\right)_{n \in N}$ is a normal sequence of open covers of $X[1,1.10]$ such that $\mathscr{U}_{1}$ refines Q 0 . Hence there exists a continuous pseudometric $d$ on $X$ that is associated with $\mathscr{W}$ such that $\left(B\left(x, 1 / 2^{3}\right)\right)_{x \in X}$ refines $\mathscr{W}[1,8.6]$. Let

$$
V=\left\{(x, y) \in X \times X \mid d(x, y)<1 / 2^{3}\right\} \text {. }
$$

Then $V$ is an element of the universal uniformity and since $V \subset W$ it follows that $W$ is also an element of the universal uniformity. The proof is now complete.

It is interesting to compare Theorem 4 to Theorem 2. In Theorem 4 we 
characterize strongly collectionwise normal in terms of a property possessed by every open cover, whereas in Theorem 2 strongly collectionwise normal was characterized in terms of a property possessed by only certain covers.

We now turn to characterizing normality in terms of weakly even covers. Recall that a cover is binary if it has exactly two elements and that normality is easily characterized in terms of conditions on every binary open cover (see [1, Theorem 11.7]).

THEOREM 5. If every binary open cover of a topological space is weakly even then $X$ is normal.

Proof. Suppose that $A_{1}$ and $A_{2}$ are disjoint closed sets. Then $थ=(X-$ $A_{1}, X-A_{2}$ ) is a binary open cover of $X$ and hence by hypothesis there exists an open symmetric neighborhood $W$ of the diagonal of $X$ such that $W^{2} \subset$ $\left(\left(X-A_{1}\right) \times\left(X-A_{1}\right)\right) \cup\left(\left(X-A_{2}\right) \times\left(X-A_{2}\right)\right)$. But then $W\left(A_{1}\right)$ and $W\left(A_{2}\right)$ are disjoint open sets containing $A_{1}$ and $A_{2}$ respectively. Therefore, $X$ is normal.

THEOREM 6. If $X$ is a topological space then the following statements are equivalent:

(1) $X$ is normal.

(2) Every binary (respectively finite, locally finite, star-finite) open cover is even.

(3) Every binary (respectively finite, locally finite, star-finite) open cover is weakly even.

Proof. Note that star finite implies locally finite implies finite implies binary. Also every even cover is weakly even and a binary open cover of a normal space is even. These observations together with Theroem 5 yield the desired result.

We now turn io even-screenable and even-expandable collections of a topological space. Our remaining results will be consequences of the following general theorem.

THEOREM 7. If $\mathcal{G}=\left(G_{\alpha}\right)_{\alpha \in I}$ is a locally finite family of open subsets of $X$ and if $\mathscr{F}=\left(F_{\alpha}\right)_{\alpha \in I}$ is a locally finite family of closed subsets of $X$ such that $F_{\alpha} \subset G_{\alpha}$ for all $\alpha \in I$ and $\bigcup_{\alpha \in I} F=\bigcup_{\alpha \in I} G$ then

(1) $\mathcal{G}$ is even-screenable and

(2) $\mathscr{F}$ is even-expandable.

Proof. Let $[I]$ be the set of all finite subsets of $I$ and for each $J \in[I]$ define

$$
A_{J}=\left(\cap\left\{G_{\alpha} \mid \alpha \in J\right\}\right) \cap\left(\cap\left\{X-F_{\alpha} \mid \alpha \notin J\right\}\right)
$$

and set $\mathscr{Q}=\left(A_{J}\right)_{J \in[I]}$. Clearly $\mathscr{Q}$ is a cover of $X$. We will show $\mathscr{Q}$ is locally finite and open.

(1) Let $A_{J} \in \mathbb{Q}$. The family $\mathscr{F}$ is a locally finite family of closed sets so $\cup\left\{F_{\alpha} \mid \alpha \notin J\right\}$ is closed. Thus $X-\cup\left\{F_{\alpha} \mid \alpha \notin J\right\}=\bigcap\left\{X-F_{\alpha} \mid \alpha \notin J\right\}$ is 
open and $A_{J}$ is the intersection of a finite number of open sets and hence open. Thus $Q$ is an open cover.

(2) Let $x \in X . \mathcal{G}$ is locally finite so there exist a neighborhood $N$ of $x$ and a finite subset $K$ of $I$ such that $N \cap G_{\alpha}=\varnothing$ if and only if $\alpha \notin K$. Let $\mathcal{K}$ be the set of all subsets of $K$. Then $\mathscr{K}$ is a finite collection of [I] and $A_{J} \cap N=\varnothing$ if $J \notin \mathscr{K}$. For if $A_{J} \cap N \neq \varnothing$ we must show $J \in \mathscr{K}$; i.e., $J \subset K$. If $\alpha \in J$ then $A_{J} \cap N \neq \varnothing$ implies $G_{\alpha} \cap N \neq \varnothing$ and, therefore, $\alpha \in K$. Thus $Q$ is a locally finite open cover of $X$.

For each $\alpha \in I$, let $H_{\alpha}=\operatorname{st}\left(F_{\alpha}, Q\right)$, set $U_{\alpha}=\left(H_{\alpha} \times H_{\alpha}\right) \cup\left(\left(X-F_{\alpha}\right) \times\right.$ $\left(X-F_{\alpha}\right)$ ) and set $U=\bigcap_{\alpha \in I} U_{\alpha}$. We assert that $U$ is a neighborhood of the diagonal of $X$ such that $U\left(F_{\alpha}\right) \subset G_{\alpha}$.

To show $U$ is a neighborhood of the diagonal of $X$ let $(x, x)$ be an element of the diagonal. Note that $H_{\alpha} \subset G_{\alpha}$ for all $\alpha \in I$ since $A_{J} \cap F_{\alpha} \neq \varnothing$ implies $\alpha \in J$ and, therefore, $A_{J} \subset G_{\alpha}$. Since $\mathcal{G}$ is locally finite $\mathscr{F}$ is locally finite and hence there exist a neighborhood $N$ of $x$ and a finite subset $K$ of $I$ such that $N \cap F=\varnothing$ if and only if $\alpha \notin K$. Let $V=(N \times N) \cap\left(\cap_{\alpha \in K} U_{\alpha}\right)$ and observe that $V$ is a neighborhood of $(x, x)$. If $\alpha \in K$ then $V \subset U_{\alpha}$ by definition of $V$. If $\alpha \notin K$ then $N \cap F=\varnothing$ so $N \subset X-F_{\alpha}$ and, therefore, $V \subset U_{\alpha}$. Thus $V \subset U_{\alpha}$ for all $\alpha \in I$ hence $V \subset U$. It follows that $U$ is a neighborhood of the diagonal of $X$. It is easily seen that $U\left(F_{\alpha}\right) \subset G_{\alpha}$.

Therefore, $\mathcal{G}$ is even-screenable and $\mathcal{F}$ is even-expandable by definition.

This result yields several very interesting facts about normal and collectionwise normal spaces. First let us observe the following.

Proposition 8. If $\mathcal{G}=\left(G_{\alpha}\right)_{\alpha \in I}$ is a cover of a topological space $X$ then (1) implies (2) implies (3).

(1) $\mathcal{G}$ is 2-even.

(2) $\mathcal{G}$ is even-shrinkable.

(3) $\mathcal{G}$ is even.

Proof. (1) implies (2). If $\mathcal{G}$ is 2-even then there exist open symmetric neighborhoods $W_{1}$ and $W_{2}$ of the diagonal of $X$ such that $W_{2}^{2} \subset W_{1}$ and $\mathscr{W}=\left(W_{1}(x)\right)_{x \in X}$ refines $\mathcal{G}$. For each $\alpha \in I$ let

$$
F_{\alpha}=\cup\left\{W_{2}(x) \mid W_{1}(x) \subset G_{\alpha}\right\} .
$$

Then $\mathscr{F}=\left(F_{\alpha}\right)_{\alpha \in I}$ is an open cover of $X$ and we assert that $W_{2}\left(F_{\alpha}\right) \subset G_{\alpha}$. If $x \in W_{2}\left(F_{\alpha}\right)$, then there exists $y \in F_{\alpha}$ such that $(x, y) \in W_{2}$. Now $y \in F_{\alpha}$ implies $y \in W_{2}(z)$ and $W_{1}(z) \subset G_{\alpha}$. But then $(x, y) \in W_{2}$ and $(y, z) \in W_{2}$ so $(x, z) \in W_{2}^{2} \subset W_{1}$ and $W_{1}(z) \subset G_{\alpha}$ implies $x \in G_{\alpha}$.

(2) implies (3). This follows from the observation that $W(x) \subset W\left(F_{\alpha}\right)$ if $x \in F_{\alpha}$ and $W$ is a neighborhood of the diagonal of $X$.

THEOREM 9. If $X$ is a topological space then the following statements are equivalent:

(1) $X$ is normal.

(2) Every binary (resp. finite, locally finite, star-finite) open cover of $X$ is even-screenable. 
Proof. (1) implies (2). If $\mathcal{G}=\left(G_{\alpha}\right)_{\alpha \in I}$ is a star-finite open cover then by a double application of Theorem 6 there exists an open cover $\mathscr{F}=\left(F_{\alpha}\right)_{\alpha \in I}$ such that cl $F_{\alpha} \subset G_{\alpha}$. Therefore, by Theorem 7, $\mathcal{G}$ is even-screenable.

(2) implies (1). Since an even-screenable cover is even, this follows from Theorem 6.

THEOREM 10. If $X$ is a topological space, then the following statements are equivalent:

(1) $X$ is collectionwise normal.

(2) If $\left(F_{\alpha}\right)_{\alpha \in I}$ is a discrete family of closed subsets of $X$, then there exists a neighborhood $W$ of the diagonal of $X$ such that $\left(W\left(F_{\alpha}\right)\right)_{\alpha \in I}$ is a pairwise disjoint family.

Proof. That (2) implies (1) is immediate, so let $\mathscr{F}=\left(F_{\alpha}\right)_{\alpha \in I}$ be a discrete family of closed sets. By hypothesis, $X$ is collectionwise normal, so there is a family $\mathcal{G}=\left(G_{\alpha}\right)_{\alpha \in I}$ of pairwise disjoint open sets such that $F_{\alpha} \subset G_{\alpha}$ for each $\alpha \in I$. Thus by Theorem 7 there is a neighborhood $W$ of the diagonal such that $W\left(F_{\alpha}\right) \subset G_{\alpha}$ for each $\alpha \in I$. Now since $\mathcal{G}$ is a pairwise disjoint family so is $\left(W\left(F_{\alpha}\right)\right)_{\alpha \in I}$.

\section{REFERENCES}

1. R. A. Alo and H. L. Shapiro, Normal topological spaces, Cambridge Univ. Press, Cambridge, 1974.

2. R. H. Bing, Metrization of topological spaces, Canad. J. Math 3 (1951), 175-186.

3. H. J. Cohen, Sur un problème de M. Dieudonné, C. R. Acad. Sci. Paris 234 (1952), 290-292.

4. H. L. Shapiro and F. A. Smith, Even covers and collectionwise normal spaces, Canad. J. Math. (to appear).

Department of Mathematics, Northern Illinois University, DeKalb, Illinois 60115

Department of Mathematics, Kent State University, Kent, Oho 44242 